Nloman 2019, 37(2), 63-69

Revista de Psicologia, Ciències de l'Eduació i de l'Esport

ISSN: $1138-3194$

CFacultat de Psicologia, Ciències de l'Educació i de l'Esport Blanquerna

Universitat Ramon Llull

(c) (1) $\$$

Y NG SA

\title{
Efectividad de la metodología Flipped Classroom en la mejora del clima social en el aula de educación primaria
}

\author{
Héctor Galindo-Domínguez \\ Universidad de Deusto
}

Recibido: 2019-4-7

Aceptado: 2019-10-2

Efectividad de la metodología Flipped Classroom en la mejora del clima social en el aula de educación primaria

Resumen. Con la introducción de las nuevas tecnologías al mundo educativo, el Flipped Classroom es una metodología que cada vez va ganando más importancia en las aulas de educación primaria. En este sentido, el objetivo de este estudio ha sido el de conocer el efecto de esta metodología sobre el clima social del aula. Para ello tomaron participaron en el estudio 852 estudiantes (Edad = 10.82; DT = .867), divididos en un grupo control (437 estudiantes) y un grupo experimental (415 estudiantes). Este último se sometió a una intervención Flipped Classroom de 2 trimestres. A ambos grupos se les pasó la escala de clima social percibido (Villa, 1992) tanto al inicio como al finalizar la intervención. Los resultados mostraron que en ninguna de las dimensiones analizadas el grupo experimental presentó diferencias significativas con respecto al grupo control, aunque sí que, en el caso del contexto interpersonal, su empeoramiento fue más leve que el del grupo control. Este fenómeno apoya la idea de que el Flipped Classroom por sí mismo no ayuda a mejorar significativamente el clima social del aula. En vista de estos resultados se discuten posibles motivos y se presentan futuras temáticas de investigación.

Palabras clave: Flipped Classroom; convivencia; educación primaria; clima social; intervención

Effectiveness of the Flipped Classroom methodology in improving the social climate in the primary education classroom

\begin{abstract}
Summary. With the arrival of new technologies to the educational world, the Flipped Classroom is a methodology that is gaining more and more importance in primary education classrooms. In this sense, the main objective of this study was been to determine the effects of this methodology on the social climate of the classroom. For this purpose, 852 students ( $A g e=10.82, S D=.867$ ), divided into a control group (437 students) and an experimental group (415 students), took part in the study. This latter group took part in a Flipped Classroom intervention lasting two terms. Both groups completed a survey on perceived social climate (Villa, 1992) at the beginning and at the end of the intervention. The results showed that the experimental group did not present significant differences with respect to the control group in any of the analysed dimensions, with the exception of the case of the interpersonal context. Here, the decrease in scores in the experimental group was milder than in the control group. These findings support the idea that the Flipped Classroom methodology by itself does not help to significantly improve the social climate of the classroom. In view of these results, possible reasons are discussed and future research topics are presented.
\end{abstract}

Keywords: Flipped Classroom; coexistence; primary education; social climate; intervention

Correspondence

Héctor Galindo-Domínguez

Psychology and Education Faculty, University of Deusto

Avenida de las universidades, 24, 48007, Bilbao (España);

688602315

hector.galindo@opendeusto.es 


\section{Introducción}

Desde hace ya unos años, en la búsqueda de la mejora de la práctica educativa, una de las variables que ha suscitado un interés creciente por parte de agencias, ministerios e instituciones educativas a nivel mundial ha sido el clima social (Thapa, Cohen, Guffey \& Higins-D'Alessandro, 2013). En algunos países, como Estados Unidos, Francia, Canadá o incluso Israel, el clima escolar es uno de los factores de calidad evaluados (López, Bilbao, Ascorra, Moya \& Morales, 2014).

En esta línea, podemos entender el clima social del aula como la percepción que cada miembro del aula tiene sobre la vida interna y diaria de la clase (Pérez, Ramos y López, 2010). Según Manota y Melendro (2016), este clima social se va consolidando sobre la base de la concreción de normas, hábitos, comportamientos y prácticas sociales manifestados a partir de las relaciones entre profesores y estudiantes.

El clima social del aula es único e intransferible; por ende, no hay dos aulas iguales, ya que este se va formando a partir de las percepciones que tienen los estudiantes de lo que ocurre dentro del aula. Estudios como el de Pérez et al. (2010) permiten mostrar que incluso los valores de clima social son diferentes entre aulas que pertenecen al mismo centro educativo.

Por otra parte, nos encontramos con el creciente interés de los estudiantes hacia el uso de las tecnologías de la información y la comunicación (Rodríguez, Martínez y Medina, 2017). Fue en este punto en el que una serie de docentes (Baker, 2000; Lage, Platt \& Treglia, 2000; Bergmann \& Sams, 2012) vieron la oportunidad de realizar un cambio en las aulas aprovechando las nuevas tecnologías que estaban al alcance de los estudiantes para fomentar los procesos de enseñanzaaprendizaje. Con esta idea surgió lo que a día de hoy se conoce como la metodología Flipped Classroom o Aula Invertida.

El Flipped Classroom es una metodología que trata de invertir los roles de la enseñanza tradicional, de manera que lo que antes se realizaba en clases, como las clases magistrales, se lleva a cabo, con esta metodología, fuera del horario lectivo - generalmente a través de vídeos educativos de corta duración-, y todo lo que antes se realizaba fuera del horario lectivo, como podían ser actividades de diferentes tipos, ahora se lleva a cabo en clase. En esta línea, las actividades que se presentan en el aula son actividades prácticas, proyectos de aprendizaje y de discusión relacionados con la resolución de problemas propuestos para los estudiantes (Lai \& Hwang, 2016).

Organizaciones internacionales como la Unesco o la OCDE han hecho especial hincapié en el desarrollo curricular de las competencias clave en educación. Estas competencias apuntan al camino que los discentes deben seguir para mejorar sus conocimientos, habilidades y actitudes para hacer frente a los retos del siglo XXI (Hwang, Lai \& Wang, 2015). Algunas de estas competencias son la comunicación, la colaboración, el pensamiento crítico, la resolución de problemas complejos o la creatividad.

En palabras de Prieto (2017), el Flipped Classroom es potencialmente beneficioso para el desarrollo de dichas competencias siempre y cuando se presente un entorno idóneo para que los estudiantes aprendan indagando sobre aspectos importantes que el docente plantee.

Dejándose guiar por el auge de las nuevas tecnologías y por los nuevos modos de innovar en el aula de educación primaria, un número cada vez más creciente de docentes han tratado de introducir esta metodología dentro de sus prácticas rutinarias y todo apunta a que este número continuará creciendo durante los próximos años (Galindo-Domínguez, 2018; Uzunboylu \& Karagözlü, 2017).

A día de hoy, la amplia mayoría de intervenciones investigadas que nos encontramos en educación primaria se han centrado mayoritariamente en conocer la efectividad del rendimiento académico o la satisfacción de la experiencia de los estudiantes, dejando de lado un abanico de variables, tal vez más interesantes, de índole personal y social, como conocer el efecto de dicha metodología sobre la gestión del tiempo, la autoeficacia, la autorregulación, la autonomía personal o las relaciones personales en el aula (Galindo-Domínguez, 2018).

Es principalmente por este motivo por el que el presente trabajo trata de arrojar algo de luz a la investigación de esta metodología, buscando en esta ocasión conocer su grado de efectividad en el aula de educación primaria sobre el clima social en esta.

\section{Método}

\section{Muestra}

La muestra completa del estudio estuvo conformada por $852($ edad $=10.82$; DT $=.867)$ estudiantes de educación primaria, divididos en dos grupos: el grupo experimental y el grupo control. Inicialmente se formó el grupo experimental con docentes que aplicaban esta metodología, con quienes se contactó mediante redes sociales y foros. A partir de los docentes que participaron en este grupo experimental, se buscaron centros de características socioeconómicas lo más similares posible, cerca del mismo entorno, para concretar el grupo control. De este modo, todos los centros se sitúan en contextos socioeconómicos medios; principalmente se trata de centros concertados y de áreas urbanas. Cada profesor va asociado a un grupo aula diferente.

El grupo experimental estuvo compuesto por 415 estudiantes $($ edad $=10.84 ;$ DT $=.817)$. Del total, 200 eran chicos y 215 eran chicas. El grupo experimental fue el encargado de realizar una intervención sobre Flipped Classroom de una duración de dos trimestres escolares, desde septiembre del 2018 hasta abril del 2019, en la asignatura que cada docente impartía a su grupo aula. En esta línea se evaluó la práctica de los docentes que utilizaron esta metodología para compro- 
Tabla 1. Indicadores ad hoc sobre el uso del Flipped Classroom en el aula de educación primaria.

\begin{tabular}{|c|c|}
\hline \multicolumn{2}{|c|}{ Flexible Environment (Entorno flexible) } \\
\hline Flexibilidad temporal y espacial & $\begin{array}{l}\text { He dejado espacios y tiempos razonables para que cada estudiante pueda visualizar los vídeos propuestos fuera del } \\
\text { colegio. }\end{array}$ \\
\hline Accesibilidad & $\begin{array}{l}\text { He hecho uso de diferentes métodos para que los estudiantes puedan acceder a los vídeos por sí mismos fuera del } \\
\text { colegio (Moodle, USB...). }\end{array}$ \\
\hline $\begin{array}{l}\text { Adaptabilidad y personalización } \\
\text { del aprendizaje }\end{array}$ & $\begin{array}{l}\text { Cuando he observado que mis estudiantes no han comprendido correctamente el contenido de un vídeo, he tratado de } \\
\text { adaptarme a ellos y he modificado el ritmo de aprendizaje. }\end{array}$ \\
\hline \multicolumn{2}{|c|}{ Learning Culture (Cultura del aprendizaje) } \\
\hline Responsabilidad & He hecho ver a mis estudiantes la importancia de realizar la tarea de casa para progresar en su proceso de aprendizaje. \\
\hline Autonomía & $\begin{array}{l}\text { Trabajar a través de este modo creo que ha permitido a mis estudiantes darse cuenta de que son capaces de trabajar y } \\
\text { aprender más por sí solos. }\end{array}$ \\
\hline Automejora & $\begin{array}{l}\text { Cuando se ha puesto en común lo visto a través de los vídeos, he permitido que mis estudiantes se diesen cuenta de sus } \\
\text { errores y pudiesen corregirlos. }\end{array}$ \\
\hline Actividades significativas & $\begin{array}{l}\text { Tras haber interiorizado el contenido de los vídeos, he propuesto actividades significativas de orden medio-superior } \\
\text { (aplicar, analizar, crear o evaluar) a mis estudiantes. }\end{array}$ \\
\hline \multicolumn{2}{|c|}{ Intentional Content (Contenido intencional) } \\
\hline Duración & La duración de los vídeos que he empleado ha sido corta (2 minutos o menos). \\
\hline Voz & En los vídeos que he utilizado la voz empleada ha sido una voz animada, no monótona. \\
\hline Humor & $\begin{array}{l}\text { Para captar mejor la atención, en momentos estratégicos del vídeo he buscado/he creado vídeos con partes donde se } \\
\text { hace uso del humor. }\end{array}$ \\
\hline Relevancia y precisión & El tema expuesto en los vídeos ha sido lo más conciso posible. \\
\hline Recordatorios & Los vídeos que empleo poseían notas informativas para aclarar algunas ideas clave explicadas. \\
\hline Herramientas complementarias & $\begin{array}{l}\text { He empleado algún tipo de herramienta complementaria al propio vídeo (Edpuzzle, Plickers...) para conocer si el } \\
\text { estudiante ha interiorizado el contenido del vídeo. }\end{array}$ \\
\hline \multicolumn{2}{|c|}{ Professional Educator (Educador profesional) } \\
\hline Interpretación del entorno & He analizado, evaluado e interpretado las notas que han obtenido mis estudiantes a través de los vídeos vistos. \\
\hline Feedback & $\begin{array}{l}\text { Tras evaluar los resultados obtenidos a través de los vídeos y las actividades de clase, he proporcionado } \\
\text { retroalimentación a cada estudiante. }\end{array}$ \\
\hline Supresión de la clase magistral & $\begin{array}{l}\text { Tras haber eliminado la parte más teórica de la clase presencial a través de los vídeos, he hecho uso de la clase magistral } \\
\text { únicamente cuando he observado dificultades severas. }\end{array}$ \\
\hline Reflexión de la práctica docente & $\begin{array}{l}\text { Sobre la base de los errores cometidos por mis estudiantes tanto en clase como en la evaluación de los vídeos, me he } \\
\text { autoevaluado y he repensado y modificado mi práctica (vídeos enviados, actividades enviadas, tareas en clase, modo de } \\
\text { proporcionar feedback...) para poder mejorar la calidad de mis clases. }\end{array}$ \\
\hline
\end{tabular}

bar que, efectivamente, lo que se aplicaba era Flipped Classroom a través de una serie de indicadores ad hoc basados en los pilares estándares del Flipped Learning (Flipped Learning Network, 2012). Estos indicadores quedan recogidos en la tabla 1.

Por otra parte, el grupo control estuvo compuesto por 437 estudiantes $($ edad $=10.81$; DT $=.912)$. Del total, 223 eran chicos y 214 eran chicas.

Ambos grupos desarrollaron las asignaturas impartidas por el docente que participaba en la intervención. La única diferencia fue que el grupo experimental aplicó la metodología Flipped Classroom junto con otras (aprendizaje cooperativo, proyectos, retos, problemas, gamificación), mientras que el grupo control empleó las mismas metodologías que el grupo experimental a excepción del Flipped Classroom.

\section{Instrumento}

Para medir el clima social del aula en educación primaria, se hizo uso de la adaptación española de la escala de clima social percibido diseñada por Villa (1992) y basada en la escala de Marjoribanks (1980).

La estructura factorial de este instrumento viene definida por cuatro factores repartidos en 28 ítems medidos en escala de Likert de 5 puntos: contexto interpersonal, contexto regulativo, contexto instruccional y contexto imaginativo. Villa (1992) los describe del siguiente modo:

Contexto interpersonal: hace referencia a la percepción de los estudiantes respecto a la cercanía y preocu- pación por parte de los profesores de sus problemas, es decir, un clima de calidad interpersonal, de amistad y confianza.

Contexto regulativo: se refiere a la percepción de los estudiantes del "calor" o severidad de las relaciones autoritarias en clase. Este contexto viene definido por la naturaleza de las relaciones autoritarias del profesor en las relaciones y en el ambiente.

Contexto instruccional: tiene relación con la percepción de los estudiantes sobre el interés o desinterés de los profesores por el aprendizaje. Se refiere a la percepción que los estudiantes tienen sobre la atención que pone el docente al proceso de enseñanza.

Contexto imaginativo: hace referencia a la percepción de los estudiantes respecto a un ambiente imaginativo y creativo donde ellos se ven estimulados a recrear y experimentar su mundo en sus propios términos o, contrariamente, perciben un clima rutinario, rígido $\mathrm{y}$ tradicional sin innovaciones.

Inicialmente, en septiembre del 2018, se prepararon los materiales necesarios para realizar el presente estudio. Al tratarse de estudiantes menores de edad, se comenzó preparando un consentimiento informado que firmaron y devolvieron a los correspondientes tutores. El pase del cuestionario se realizó vía online en horario lectivo en septiembre del 2018 y en abril del 2019. El tiempo que dispusieron en ambas ocasiones para responder a los cuestionarios fue de unos $40 \mathrm{mi}-$ nutos por sesión.

Cabe destacar que todos los datos recogidos cumplieron los criterios de privacidad y anonimato de los 
Tabla 2. Correlaciones entre las principales dimensiones en la fase pre y post $(n=852)$.

\begin{tabular}{|c|c|c|c|c|c|c|c|c|c|}
\hline & & \multicolumn{4}{|c|}{ Fase PRE $(\alpha=.884)$} & \multicolumn{4}{|c|}{ Fase POST $(\alpha=.913)$} \\
\hline & & Int & Reg & Inst & Ima & Int & Reg & Inst & Ima \\
\hline \multirow[t]{4}{*}{ Fase Pre } & Int & $(.732)$ & .328 & .599 & .680 & .168 & .190 & .326 & .403 \\
\hline & Reg & & $(.717)$ & .526 & .418 & .124 & .512 & .336 & .292 \\
\hline & Inst & & & $(.695)$ & .696 & .151 & .341 & .471 & .461 \\
\hline & Ima & & & & $(.766)$ & .180 & .232 & .412 & .514 \\
\hline \multirow[t]{4}{*}{ Pase Post } & Int & & & & & $(.815)$ & .156 & .211 & .210 \\
\hline & Reg & & & & & & $(.774)$ & .552 & .437 \\
\hline & Inst & & & & & & & $(.768)$ & .755 \\
\hline & Ima & & & & & & & & $(.811)$ \\
\hline
\end{tabular}

Nota: Int: contexto interpersonal; Reg: contexto regulativo; Inst: contexto instruccional; Ima: contexto imaginativo. ${ }^{*} p<.05$; ${ }^{* *} p<.000$. Se muestran los valores alfa de Cronbach en la diagonal principal de la matriz.

Tabla 3. Prueba T para cada dimensión, grupo y tiempo $(\mathrm{n}=852)$.

\begin{tabular}{|c|c|c|c|c|c|c|}
\hline \multirow[t]{3}{*}{ Dimensión } & \multirow[t]{3}{*}{ Grupo } & \multicolumn{4}{|c|}{ Tiempo } & \multirow[t]{2}{*}{ Prueba $t$} \\
\hline & & \multicolumn{2}{|c|}{ Fase Pre } & \multicolumn{2}{|c|}{ Fase Post } & \\
\hline & & $\bar{\chi}$ & DT & $\bar{\chi}$ & DT & $\mathrm{p}$ \\
\hline \multirow[t]{2}{*}{ Contexto interpersonal } & Experimental & 3.94 & .756 & 3.89 & .560 & .270 \\
\hline & Control & 3.98 & .719 & 3.84 & .536 & .000 \\
\hline \multirow[t]{2}{*}{ Contexto regulativo } & Experimental & 3.74 & .805 & 3.78 & .851 & .351 \\
\hline & Control & 3.76 & .784 & 3.79 & .866 & .359 \\
\hline \multirow[t]{2}{*}{ Contexto instruccional } & Experimental & 4.42 & .575 & 4.40 & .611 & .640 \\
\hline & Control & 4.40 & .608 & 4.39 & .674 & .698 \\
\hline \multirow[t]{2}{*}{ Contexto imaginativo } & Experimental & 4.24 & .656 & 4.20 & .696 & .220 \\
\hline & Control & 4.17 & .688 & 4.15 & .772 & .430 \\
\hline
\end{tabular}

estudiantes. Esto fue posible a través de un sistema de códigos que se acordó entre investigador y docentes.

\section{Análisis de datos}

Para analizar los datos obtenidos del cuestionario se empleó SPSS Statistics 24. Dentro del software se hizo uso de una serie de diferentes análisis.

Se comenzó estudiando las correlaciones entre los diferentes constructos y la fiabilidad del instrumento usado. Se continuó aplicando una T de Student para muestras relacionadas en aras de conocer si, para alguno de los dos grupos, el factor tiempo tuvo influencia en el clima social. Se finalizó realizando una ANOVA de medidas repetidas para conocer especialmente la interacción entre tiempo y grupo.

\section{Resultados}

Se comenzó inspeccionando los datos a través de un análisis correlacional de las dimensiones principales, tanto en la fase pre como en la fase post.

Como puede observarse en la tabla 2, tanto en la fase pre $(r=.328 ; p=.000$ a $r=.696 ; p=.000)$ como en la fase post $(r=.156 ; p=.000$ a $r=.755 ; p=.000)$ la correlación entre todas las dimensiones resultó estadísticamente significativa.

Es interesante destacar que también existe una correlación significativa de cada dimensión entre su fase pre y su fase post $(r=.168 ; p=.000$ a $r=.514 ; p$ $=.000$ ).

Resulta lógico que haya correlaciones muy elevadas, por ejemplo, la existente entre el contexto instructivo y el contexto imaginativo $(r=.755 ; p=.000)$, pues el interés positivo de los docentes hacia la práctica educativa se espera que correlacione muy positivamente, como es el caso, con la capacidad para crear un ambiente creativo, imaginativo e innovador; o entre el contexto interpersonal y el contexto imaginativo $(r=$ $.680 ; p=.000$ ), pues se espera que un clima de mayor cercanía y apoyo a los estudiantes ayude a que se pueda crear un ambiente más innovador, centrado en el estudiante.

Por su parte, se estudió la fiabilidad de cada dimensión, encontrando valores satisfactorios de consistencia interna, yendo estos en la fase pre desde $\alpha=.695$ hasta .766 y en la fase post desde $\alpha=.768$ hasta $\alpha=.815$.

Viendo que estos análisis preliminares resultaron satisfactorios y con el fin de conocer si existían diferencias significativas entre el momento inicial y el momento final de cada grupo, se realizó una prueba $t$ de Student para muestras relacionadas. En este punto únicamente se pretendía mostrar información sobre las posibles diferencias de cada grupo y en cada momento respecto a cada dimensión. Toda esta información se recoge en la tabla 3.

De estos datos, se observó que únicamente existían diferencias en el empeoramiento del grupo control en cuanto al contexto interpersonal $(p=.000)$.

A continuación se analizó la interacción de cada grupo y momento en cada dimensión. Para este fin se hizo uso de una ANOVA de medidas repetidas, considerando como variables intrasujetos la fase pre y post de cada dimensión y considerando como variable intersujetos el grupo al que pertenecía cada sujeto (control o experimental). De igual modo, cabe mencionar que se compararon los efectos principales con el ajuste de intervalo de confianza de Bonferroni. 


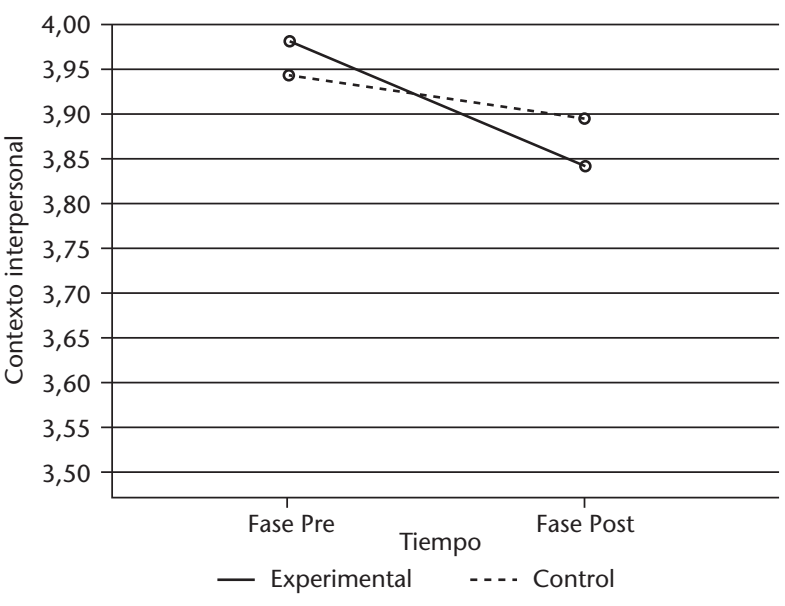

Figura 1. Valores del contexto interpersonal en función del grupo y tiempo $(n=852)$.

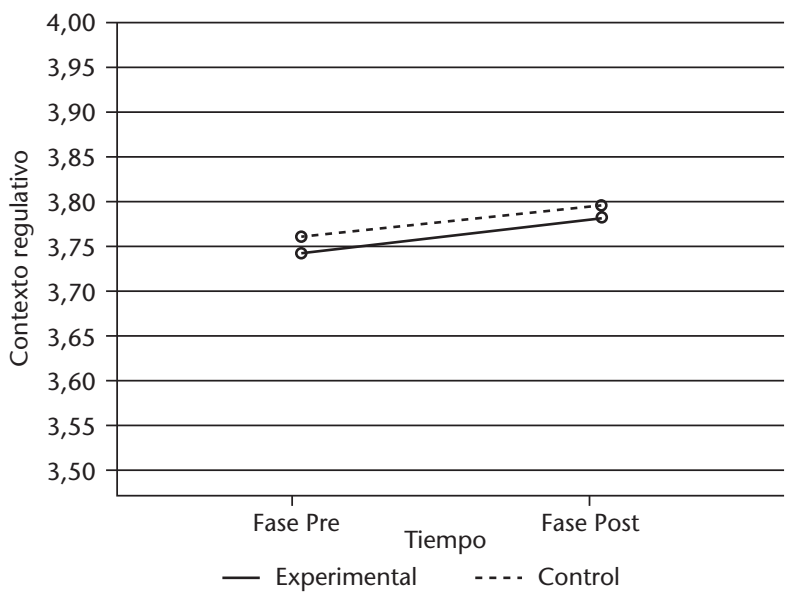

Figura 2. Valores del contexto regulativo en función del grupo y tiempo $(n=852)$.

En primer lugar, respecto al contexto interpersonal, en la figura 1 queda ilustrado el efecto de la intervención en ambos grupos. En este caso, la interacción entre tiempo y grupo obtuvo un valor $F=2.49, p=.144, \eta_{p}{ }^{2}=$ .003 , lo que supuso que no existían diferencias significativas entre los grupos tras la aplicación de la intervención, aunque como ya comentamos con anterioridad sí que la diferencia entre ambos momentos de la intervención fue significativa en el caso del grupo control.

En segundo lugar, se analizó el contexto regulativo. Como se muestra en el gráfico 2, la interacción, nuevamente, entre tiempo y grupo obtuvo un valor $F=$ $.006, p=.940, \eta_{p}^{2}=.000$, siendo este valor no significativo, por lo que las diferencias entre ambos grupos en función de la intervención no resultaron notorias.

Seguidamente, como se muestra en la figura 3, se analizó el contexto instruccional. Siguiendo con el mismo procedimiento, la interacción entre tiempo y grupo para esta dimensión fue de $F=.007, p=.932, \eta_{p}{ }^{2}$ $=.000$. Este valor llevó una vez más a la conclusión de que la aplicación del Flipped Classroom no suponía ningún efecto especial con respecto al uso de otras metodologías.

Finalmente, respecto al contexto imaginativo, en la figura 4 se ilustra el efecto de la intervención en

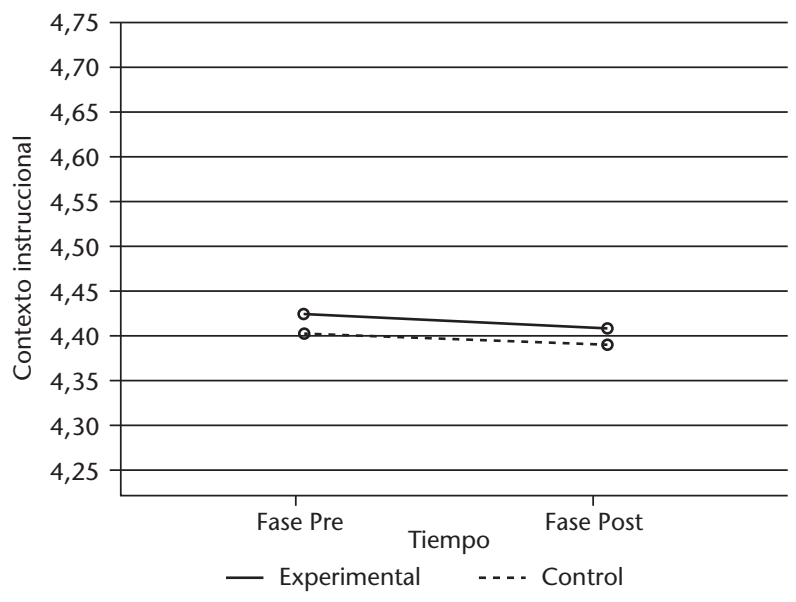

Figura 3. Valores del contexto instruccional en función del grupo y tiempo $(n=852)$.

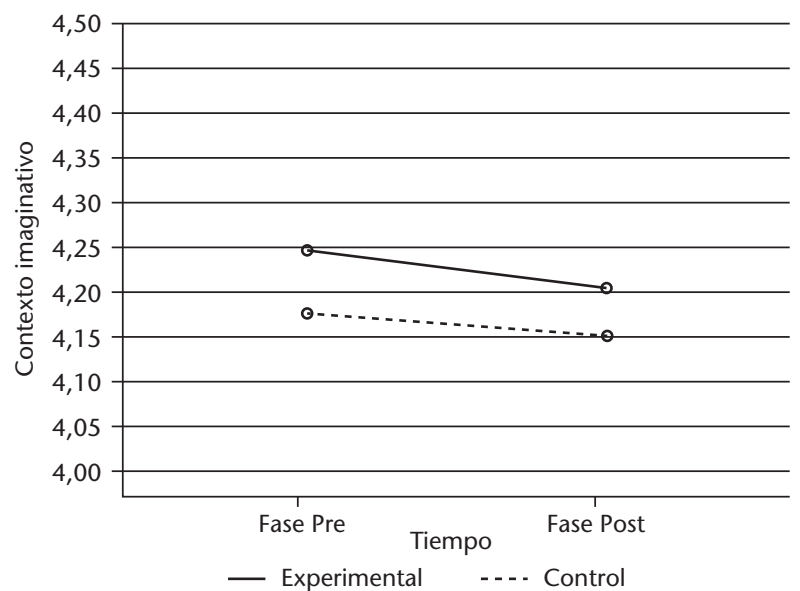

Figura 4. Valores del contexto imaginativo en función del grupo y tiempo $(n=852)$.

ambos grupos. En este caso, la interacción entre tiempo y grupo obtuvo un valor $F=.131, p=.718, \eta_{p}^{2}=$ .000 , lo que implicaba por última vez que no existían diferencias significativas entre los grupos tras la aplicación de la intervención.

Por lo tanto, se pudo observar que, tras la aplicación de la intervención Flipped Classroom en el aula de educación primaria, no mejoró ninguna de las dimensiones estudiadas; o dicho con otras palabras, el uso de la metodología Flipped Classroom no sirvió más que otras metodologías como modo de mejora del clima social en el aula.

\section{Discusión}

El objetivo principal del presente trabajo ha sido analizar la efectividad de la metodología Flipped Classroom sobre el clima social del aula de educación primaria. Para este fin, se realizaron una serie de análisis bivariados y multivariados partiendo de una muestra de estudiantes de educación primaria que formaron parte de un grupo control y un grupo experimental. Estos últimos tomaron parte de una intervención en la que se aplicaba de manera consistente la metodología Flipped Classroom. 
Los hallazgos obtenidos en el presente trabajo mostraron que la intervención del Flipped Classroom para la mejora del clima social del aula de educación primaria no fue estadísticamente efectiva en ninguna de sus dimensiones.

Resultó curioso que, en todas las dimensiones del clima social, los estudiantes que tomaron parte en el grupo experimental partiesen de valores superiores a sus coetáneos del grupo control. Esperando que la intervención fuese efectiva y los valores mejorasen, el efecto obtenido fue totalmente contrario. En todos los casos, los estudiantes de ambos grupos percibieron el clima social de su aula peor respecto a cuando comenzaron el curso escolar.

Tanto en el caso del contexto regulativo $(p=.940)$ como instruccional $(p=.932)$ e imaginativo $(p=.718)$, los resultados se mostraron lejos de ser significativos. Este fenómeno se observó claramente en las figuras 2, 3 y 4 , en las que las líneas respectivas al grupo control y experimental avanzan de manera casi paralela.

En relación con el contexto interpersonal, aunque es cierto que los resultados tampoco fueron estadísticamente significativos $(p=.144)$, sí se observan las diferencias entre el grupo control y el grupo experimental; así, se aprecia que el grupo control valoró de manera más negativa las relaciones autoritarias del profesor en el ambiente que el grupo que tomó parte en la intervención.

En vista de estos resultados, se cree que existe una serie de factores más importantes que únicamente una metodología empleada en clase para mejorar el clima social del aula de educación primaria, factores tales como la motivación que sea capaz de crear el docente, la libertad de expresión, la ayuda mutua ante problemas... (Afari, Aldridge, Barry, Fraser \& Swekhine, 2013; Ju-Sen \& Chauyun, 2014; Magen-Nagar \& Azuly, 2016). En esta línea, el estudio apoya la idea de que no existe ninguna metodología que funcione como panacea, al menos en educación primaria.

Se piensa que, en educación primaria, los docentes están haciendo cada vez más un esfuerzo por pasar de metodologías más tradicionales a otras en las que el estudiante se sitúa en el centro del proceso de aprendizaje. Es por ello por lo que en el presente estudio, a pesar de que un grupo haya aplicado el Flipped Classroom y el otro no, hay una serie de metodologías activas, como el aprendizaje cooperativo, aplicadas en ambos grupos que es posible que hayan afectado a ambos grupos en la fluctuación de los valores del clima social.

Tal vez el Flipped Classroom pueda servir para mejorar el clima social del aula, más que en educación primaria, en las aulas universitarias, donde, entre otros motivos, por el gran número de estudiantes a menudo se tiende a priorizar la clase magistral frente a otras metodologías. Es aquí tal vez donde el Flipped Classroom, junto con otras metodologías activas, puede mejorar el clima frente a esa clase tradicional en la que los estudiantes apenas interaccionan tanto entre ellos como con el profesor.
Respecto a los puntos mejorables del presente estudio, resulta importante destacar cuáles son las limitaciones más significativas.

En primer lugar, es complicado achacar completamente los resultados obtenidos al uso de la metodología Flipped Classroom, cuando lo cierto es que, al igual que en cualquier cuasi experimento, existe una serie casi infinita de factores en los que el sujeto está inmerso que conllevan que, dependiendo de la ocasión, este puntúe el clima social de una manera u otra.

Seguidamente, tal y como ya se ha comentado, a pesar de que el grupo experimental hiciese uso de la metodología Flipped Classroom, en educación primaria resulta imposible aislar una serie de técnicas y metodologías que se han podido compartir en ambos grupos, como aprendizaje cooperativo, aprendizaje basado en proyectos, aprendizaje basado en retos y problemas, rutinas de pensamiento, etc. En educación primaria resulta prácticamente imposible realizar un cuasi experimento con un grupo control que aplique exclusivamente una metodología tradicional, de forma que las técnicas y metodologías queden claramente diferenciadas.

Por último, la muestra empleada es algo pequeña, aunque en esta línea cabe mencionar que no resulta nada fácil hallar docentes que apliquen esta metodología de manera tan continua para preparar una intervención de estas características.

En un mundo en el que cada vez se está intentando dar más importancia no solo a lo académico sino también a lo social y a lo afectivo, es de subrayar que futuros estudios podrían continuar analizando el efecto de esta metodología en las aulas de educación primaria desde diferentes constructos sociales y emocionales, tales como la orientación motivacional, el autoconcepto, el grado de autonomía, la autorregulación... Esta futura línea de estudios ya ha sido recomendada en metaanálisis previos, en los que se observó que el constructo por defecto de los estudios que analizan la efectividad del Flipped Classroom en educación primaria es únicamente el rendimiento académico (GalindoDomínguez, 2018). El camino que queda por recorrer en este ámbito aún es largo.

\section{Declaración de divulgación del autor}

No existen intereses en conflicto.

\section{Referencias}

Afari, E., Aldrige, J. M., Fraser J. B., \& Swe Khine, M. (2013). Students' Perceptions of the Learning Environment and Attitudes in Game-Based Mathematics Classrooms. Learning Environments Research, 16, 131150. http://dx.doi.org/10.1007/s10984-012-9122-6

Baker, J. W. (2000). The "Classroom Flip": Using web course management tools to become the guide by the side. En J. A. Chambers (Ed.), 11th International Conference on College Teaching and Learning (pp. 9-17). Jacksonville, Florida: Florida Community College. 
Bergmann, J. \& Sams, A. (2012). Flip your Classroom: Reach Every Student in Every Class Every day. Washington, EEUU: ISTE.

Uzunboylu, H. \& Karagözlü, D. (2017). The emerging trend of the flipped classroom: A content analysis of published articles between 2010 and 2015. Revista de Educación a distancia, 54, 1-13. http://dx.doi. org/10.6018/red/54/4

Galindo-Domínguez, H. (2018). Un meta-análisis de la metodología Flipped Classroom en el aula de Educación Primaria. EDUTEC, revista electrónica de tecnología educativa, 63, 73-85. https://doi.org/10.21556/ edutec.2018.63.983

Lai, C.L. \& Hwang, G.J. (2016). A self-regulated flipped classroom approach to improving students' learning performance in a mathematics course. Computers \& Education, 100, 126-140. https://doi.org/10.1016/j. compedu.2016.05.006

Hwang, G.J., Lai, C.L. \& Wang, S.Y. (2015). Seamless flipped learning. A mobile technology-enhaced flipped classroom with effective learning strategies. Journal of Computers in Education, 2 (4), 449-473. https://doi.org/10.1007/s40692-015-0043-0

Prieto, A. (2017). Flipped Learning. Aplicar el modelo de Aprendizaje Inverso. Madrid, España: Narcea.

Flipped Learning Network (2014). The Four Pilars of F-L-I-P. Flipped Learning. Recuperado de https:// flippedlearning.org/wp-content/uploads/2016/07/ FLIP_handout_FNL_Web.pdf

Ju-Sen, L., \& Chaoyun, L. (2014). The Perceived Influence of Learning Environment on Design Student Imagination. International Journal of Learning, Teaching and Educational Research, 2, 124-136.

Lage, M.J. Platt, G.J. \& Treglia, M. (2000). Inverting the Classroom: A gateway to creating an inclusive learning environment. Journal of Economic Education, 31 (1), 30-43. https://www.jstor.org/stable/1183338
López, V., Bilbao, M. A., Ascorra, P., Moya, I. \& Morales, M. (2014). Escala de clima escolar: Adaptación al español y validación en estudiantes chilenos. Universitas Psychologica, 13 (3), 1111-1122. https://psycnet. apa.org/doi/10.11144/Javeriana.UPSY13-3.ecea

Magen-Nagar, N. \& Azuly, D. (2016). The contribution of School climate and Teaching Quality to the improvement of Learning achievements, According to an external evaluation system. Creative Education, 7, 1773-1784. http://dx.doi.org/10.4236/ ce. 2016.713181

Marjoribanks, K. (1980). School Environment Scale. Adelaide, Australia: Jan Press.

Manota, M.A. \& Melendro, M. (2016). Clima de aula y buenas prácticas docentes con adolescentes vulnerables: más allá de los contenidos académicos. Contextos Educativos, 19, 55-74. http://dx.doi.org/10.18172/ con. 2756

Pérez, A., Ramos, G. \& López, E. (2010). Clima social aula: percepción diferenciada de los alumnos de educación secundaria obligatoria. Cultura y Educación, 22 (3), $259-281$. http://dx.doi. org/10.1174/113564010804932184

Rodríguez, B. P., Martínez, R. D. \& Medina, C. P. (2017). Uso de las tecnologías de la información y la comunicación (TIC) como recurso didáctico promotor de convivencia escolar. Revista Espacios, 38 (30), 24.

Thapa, A., Cohen, J., Guffey, S. \& Higgins-D’Alessandro, H. (2013). A review of school climate research. Review of Educational Research, 83 (3), 357-385. https://doi. org/10.3102\%2F0034654313483907

Villa, A. (1992). El Clima Escolar en los centros de EGB de Vizcaya, Medido a través del S.E.S. En A. Villa \& L. M. Villar (Coords.), Clima organizativo y de aula. Teorías, modelos e instrumentos de medida (pp. 119134). Vitoria-Gasteiz, España: Servicio Central de Publicaciones del Gobierno Vasco. 